\title{
Teaching of Mathematical Analysis or Calculus Based on the Thinking of Calculus
}

\begin{abstract}
Wei Wang*
School of Mathematics, Renmin University of China

*Corresponding author. Email: wwei@ruc.edu.cn

ABSTRACT

Mathematical analysis or calculus is one of the core courses of mathematics and many related disciplines. For students to lay solid foundations and to have better developments in their future careers, teaching of mathematical analysis plays an important role in mathematics and related disciplines. Unfortunately, many students feel that it is difficult in the understanding and learning of the subject. Based on many years of the teaching experiences, in this paper, we put forward the new idea that the teaching of mathematical analysis can be carried out by using the thinking of calculus. The essence of the method is to carry out the teaching and learning of mathematical analysis or calculus with a simple and efficient way. Some suggestions on the teaching and learning of the subject are also provided.
\end{abstract}

Keywords: Teaching reformation, Mathematical analysis, Calculus thinking, Automatic method.

\section{INTRODUCTION}

Calculus or mathematical analysis is one of the basic courses of mathematics, and it is also a basic course for many other majors. It plays an important role in cultivating students' mathematical professionalism and mastering scientific and efficient calculation methods.

However, in the teaching procedures of calculus or mathematical analysis, it is generally believed that learning of mathematical analysis or calculus is a very difficult thing. I was very impressed by a very serious student who had studied calculus for a year. When she was asked what she felt about the course, her answer was that it is nothing but difficulty, or it is more difficulty than difficulty. In addition, a considerable number of students fail in this course each year.

During the mid-autumn festival in 2020, the mooncakes sold by the University of Science and Technology of China (USTC) take some of the courses which are difficulty to learn, such as mathematical analysis, as the object of moon cakes. The moon cake packaging sent to students by USTC the year 2020 is full of creativity. The packaging box of mooncake is covered with the textbooks of Astrophysics, the Course of Mathematical Analysis, Introduction to Quantum Mechanics and other textbooks, which are marked with 'Excellent Textbooks of China University of Science and Technology'. The small package also has the words 'for you'. The official micro-blog of USTC also wishes that the students will never fail after eating the cake. It indicates that mathematical analysis is also a difficult course for students of USTC.

A foreign colleague lamented that it is not easy to teach multiple variable calculus, because students do not have enough motivation. And it will be easier to teach the multi-variable analysis only when students knowing the applications.

Then, why should the students learn this course well? How can we carry out the teaching of calculus and mathematical analysis, and how can we simplify the problem of teaching and learning? Combining with ourselves experiences in teaching of calculus or mathematical analysis for many years, in this paper, we put forward the viewpoint of using the thinking of calculus to carry out the teaching of calculus or mathematical analysis. First of all, our viewpoint is that mathematical analysis is necessary for almost all majors, and it should be taught or learned seriously. At the same time, our teaching should adopt the idea of calculus, i.e., we should try our best to translate some of the complex calculations into simple ones, and to turn the problem of complex learning processes into easy learning processes.

The organizing of the paper is as follows. In section 2 , we will concern about the significances and the roles of mathematical analysis; In section 3, the viewpoints 
on the contents of mathematical analysis is discussed; In section 4, the reasons on the difficulty of learning calculus or mathematical analysis are pointed out; In section 5, to learn mathematics well, some suggestions are provided. Followed by some conclusions.

\section{THE VIEWPOINTS ON THE FUNCTIONS AND NECESSITIES OF CALCULUS OR MATHEMATICAL ANALYSIS}

From the basic viewpoint on the division of higher mathematics and elementary mathematics, mathematical analysis belongs to the higher mathematics. It is the development of elementary mathematics. Elementary mathematics concern about the mathematics of studying constants, and mathematical analysis concerns about the science of variables. Therefore, mathematical analysis provides the mathematical or scientific methods to describe the characteristics on changing of variables [1].

It is precisely because of such viewpoint that the subject of mathematical analysis is a foundation of various disciplines. Because dialectics tells us that all things or materials are always in changing, change is absolutely, and invariance is relatively. Any subject is related with the problem of changing, not only scientific subjects, but also the subjects on society, humanity and culture. How can we describe the laws or rules of changing? It is inseparable from the methods provided by mathematical analysis. In other words, mathematical analysis solves the important problems in philosophy. That is to say, it provides the measurement methods of changing. Especially, the differential or derivative solves the problem of changing speeds, and the integral solves the problem of cumulative effects on changing.

It's not only the students from mathematical majors but also for students from many other majors, who need to learn this course well. For example, in computer applications, information processing is a very important aspect. The basis methods of information processing come from mathematical analysis. If you are engaged in $\mathrm{CAD}$ or $3 \mathrm{D}$ modelling, the methods in multivariate calculus are necessary and more useful. If you are engaged in information management, the characteristics of information flow can be illustrated by the curve area, and if you concern about the economic management and resource management, you are also inseparable from mathematical analysis [5]. And, if you want to be effective in financial analysis in the future, you'll have to learn stochastic analysis, which is the extension of the methods or thinking of calculus. And mathematical analysis is also the basis of the follow-up courses of mathematics, such as, ordinary differential equations, real analysis, complex analysis, probability and statistics, which are all based on the knowledge of calculus.

\section{THE ESSENTIAL IDEAS OF CALCULUS OR MATHEMATICAL ANALYSIS}

Calculus is considered to be one of the greatest inventions in the history of human science. It is the discovery on the idea of calculus that make the scientific calculation effectively and simply [2]. As mentioned above, the basic problem of calculus is to study the methodology of solving the change of things. Therefore, it takes a series of functions as its objects. It studies the change rate or cumulative effect of the change of one variable functions at first, and then, they learn multiple variable functions. So, the subject mainly includes elementary functions and corresponding functions.

In order to solve the problem of calculus, the method of the limit theory was introduced [3]. It is the introduction of limit method that we can understand 'infinity' from 'finiteness', understand 'variable or change' from 'constant', and understand 'qualitative change' from 'quantitative change', and so on. That is the essential features of mathematical analysis, i.e., to realize the complex calculation from simple calculation.

It is the introduction of differential or derivative that makes the calculation of the increment of a complex function becomes a linear function of independent variable. In particular, differential is to solve the complex nonlinear calculation by linear 'approximation' (main component) plus infinitesimal quantity. It is because of the characteristics that make the calculation for the area or volume of unusual complex geometry simplify with efficient calculation of calculus [4].

It is because of the functions of limit that determine the structure of mathematical analysis. The whole course is composed by the concepts and properties of the limit, continuity, differentiability and integrable of a series of functions. And the fundamental part of limit theory is the theory of real numbers.

It is the establishment of the relationship between derivative and integral, i.e., Newton-Leibniz formula, that makes the analysis of the boundary situation can be reflected by the internal situation.

So, from the perspective for the majors of mathematics, it is based on real number theory, limit theory, continuity, differentiability and integrable. Each section has its corresponding concepts, properties and algorithms. From the perspective of applications, the theory on real numbers can be ignored, that is to say, the students can pay more attention to the concepts or methods of limit, continuity, differentiability and integrable without the proof of the main theories [6].

\section{WHY DO STUDENTS FEEL DIFFICULTIES FOR THE LEARNING OF MATHEMATICAL ANALYSIS?}

Why do students feel the difficulty on the learning of 
mathematical analysis or calculus? From the viewpoint of ourselves, there are some aspects affecting the performance of teaching and learning.

First of all, it relies on the serious lack of the understanding to the necessary or/and the backgrounds of mathematical analysis. The reasons why we usually feel difficult are that we don't know why to learn. Usually, the mathematical analysis is presented to the students not by the usefulness, but by the abstraction and a lot of mathematical skills. Meanwhile, the teaching of mathematical analysis usually performed by the scholars or professors who are with deep basics of mathematics, they are familiar with the way of abstract thinking of mathematics. They may or may not also understand the idea or the backgrounds behind the concepts of mathematics. They cannot tell the real backgrounds on some of the mathematical thinking, or what are the reasons which we can learn from the mathematics.

Secondly, the difficulty of mathematic analysis lies in its abstractness. Of course, this does not mean that mathematical problems and methods are purely mathematicians' business. They come from real life and production. On the other hand, textbooks often contain mainly a lot of abstract concepts, signs and skills without much more materials on the applications.

Many abstract concepts in mathematics come from real phenomena, and they are the embodiment of the most essential characteristics. For example, particles suspended in the liquid to do irregular motion is called Brownian motion. The mathematical abstraction of this motion is called Wiener process. Now, people use Brownian motion to approximately describe the changing law of the stock markets. This is one example of the important roles of mathematics. Without mathematical abstraction, it may be difficult to relate the motion of particles in liquid with the fluctuation law of stock markets. This is mathematics, i.e., it is a highly abstract science. It is this abstraction that enables people to see the essence of the problem through phenomena. It is precisely such kind of features that we can equate the oil exploration of the earth with the medical diagnosis of the disease source. Maybe the two aspects have the characteristics of 'isomorphism'. It is precisely because of this feature that we can get a breakthrough in another field by 'transplanting' under the inspiration of research methods in other fields [8].

Thirdly, the difficulty also depends on whether the basics of mathematics is solid or not. Mathematics is the subject needs strong ability of logical reasoning. The former knowledge will be important for the studying of follow-up contents. For example, many of the ideas and methods related with multi-variable calculus are based on the ones from single variable calculus. So, if students don't have a firmly grasp of the methods at the beginning, the consequences will be the ones that the students cannot understand what the teachers said later, or that the students have no idea on what we will discuss.

Maybe all of these reasons lead to students the impression of mathematical analysis that it is difficulty or more difficulty than nothing.

However, what is the inspiration for students that mathematical analysis bringing about? We really hope that mathematical analysis can tell them that calculus can make the complex calculating more easily, for example, the calculating of the area or volume for some unusual objects. And from the relationship between differential and integration, i.e., the Newton-Leibniz formula, we can understand that the cultivation of small things can bring remarkable/serious results, not only for our behaviours on the learning or actions for doing things, but also for our healthy. It is almost the same that we can learn from the aphorism, i.e., don't do anything small if it is evil, but do something small if it is good. The methods in calculus or mathematical analysis are also the scientific expressions of some well-known aphorism, such as, Rome is not built in a day.

\section{HOW CAN WE LEARN MATHEMATICAL ANALYSIS WELL?}

Mathematics is an integration composed by some abstract concepts, properties and algorithms. For example, geometry is a subject based on an axiomatic system. Similarly, probability theory is based on certain axiomatic system, and topology is embodied by some axiomatic system. Therefore, to learn mathematics well, we need to understand these axioms and the deduces based on them. Just as we need to learn the rules of the game early when playing chess or playing games. It will be easier to learn mathematical analysis or calculus by remembering the concepts and the related rules at first.

According to the analysis above, the core of mathematical analysis has its basic framework. Each part of the framework has some concepts, properties and algorithms. So, as a student, mastering the concepts of limit, continuity, derivative and integral for elementary functions, and understand them better. Even if you don't understand them, you should recite them at first, and then try to use them by doing some exercises. Only in this way, you can cultivate the self-confidence on the learning.

Therefore, to learn mathematics well, we should understand the original and development of these 'rules'. To speak back, just as the learning of English we must remember the grammar and the necessary number of words, we should remember the concepts and some algorithms at least, including some necessary skills in mathematical analysis. That is to say, we can learn by grasping the main algorithms of the subject, 
getting rid of some details and proofs. Then we do not be intimidated by some unusual skills.

I usually teach the third part of mathematical analysis. Many contents in the third part of mathematical analysis are parallel to the first part of mathematical analysis. The main considerations of my first class are to solve the problem of why and how to learn, because some students don't know what should they learn in mathematical analysis even having one year studying. Therefore, for each new problem or concept, we should always remind the students of the original problems and methods, and what is the difference in the multi-variable analysis. Once again, many calculations in the section three are based on the knowledge and conclusions of that in the section one. Therefore, the conclusions and methods of the section three are divided into the ones that should be memorized as common sense. Of course, it is the best to do so based on understanding.

To learn mathematical analysis or calculus well, we must overcome the fear of difficulties. Some students say that their foundations in middle school are not solid. As long as we understand the 'rules' in mathematical analysis, we can learn them well. We wish all the students can learn mathematical analysis well and lay a good foundation for their future development.

In addition, when learning mathematical analysis, it should be noted that some of the analysis and proof methods, such as constructing auxiliary functions, and proving convergence, were accumulated for several centuries of exploration. Therefore, we should recite the common analysis and proof methods or skills at first. This is the suggestion we got when consulting some great mathematicians from the Chinese Academy of Sciences. After that, we can solve the problem with proper imitation.

Finally, we must recognize that learning is a hard work, certain time paid is necessary. According to the statistics of foreign scholars, the ratio used for learning of mathematical analysis is $4: 1$. That is to say, if we learn one hour in class, and three times more time is needed for previewing, reviewing and doing homework.

The viewpoints above are some of our experiences, just for your reference only. We hope that all students can learn mathematical analysis or calculus easily, and they can grasp the idea effectively, and they will have a better future in their careers.

From the viewpoint of applications, the automatic method for calculating the derivatives can be realized by using tracking-differentiators [7]. With the development of artificial intelligence, we can have more chances to simplify the method for complex calculations.

\section{CONCLUSIONS}

Mathematical analysis or calculus is one of the key courses of mathematics and many related subjects.
Teaching of mathematical analysis plays an important role in mathematics and related disciplines, but many students feel that it is difficult for the learning. On the basis of the author's teaching experience for many years, this paper puts forward the viewpoints that we can teach of mathematical analysis based on the idea of calculus. Its essence is to carry out the teaching and learning of mathematical analysis with a simple and efficient way.

\section{ACKNOWLEDGMENTS}

The authors would like to thank the financial support from the 'Financial Calculation and Digital Engineering'-the Engineering Research Centre of Ministry of Education of China.

\section{REFERENCES}

[1] A. D. Alexander, et al., Mathematics: Its Contents, Methods and Significance, vol. I, CCCP, 1956. (Translated into Chinese by Xiaoli Sun, et al., published by Science Press of China, 1984.)

[2] M. Kline, Mathematical Thought: From Ancient to Modern Times, vol. I, Oxford Univ. Press, New York, 1972. (Translated into Chinese by Lijing Zhang, et al., published by Shanghai Science and Technology Press, 1981.)

[3] L. Garding, Encounter with Mathematics, Springer-Verlag, 1977. (Translated into Chinese by Zuoxuan $\mathrm{Hu}$, published by Science Press of China, 1984.)

[4] G.Z. Ouyang, etc., Mathematical Analysis, Fourth edition, Higher Education Press of China, 2017.

[5] W. Wang, Some considerations on mathematical statistics for dealing with big data from the viewpoint of educational innovation, Proc. of 2016 2nd International Conference on Management Science and Innovative Education (MSIE 2016), Advances in Social Science, Education and Humanities Research, vol.60, pp. 465-469, 2016.

[6] W. Wang, The contents of some mathematical courses need to be improved: a viewpoint for education reformation, Advances in Social Science, Education and Humanities, vol. 28, pp. 382-384, 2015.

[7] J.Q. Han and W. Wang, Nonlinear trackingdifferentiators, Systems Science and Mathematical Science, vol. 14, no.2, pp. 177-183, 1994.

[8] W. Wang, Mathematics is not just an abstract subject but an economic and effective methodology: A viewpoint for education reformation, in: Deyao Tan (Eds.), Engineering Technology, Engineering Education and Engineering Management, CRC Press, pp. 151$154,2015$. 